\title{
Effect of airborne-particle abrasion and, acid and alkaline treatments on shear bond strength of dental zirconia
}

\author{
Blanca I. FLORES-FERREYRA ${ }^{1}$, Rogelio J. SCOUGALL-VILCHIS ${ }^{1}$, Ulises VELAZQUEZ-ENRIQUEZ1', \\ Rene GARCIA-CONTRERAS ${ }^{2}$, Lizzeth AGUILLON-SOL ${ }^{1}$ and Oscar F. OLEA-MEJIA ${ }^{3}$
${ }^{1}$ Dental Research Center "Dr. Keisaburo Miyata", School of Dentistry, Autonomous University of Mexico State, Av. Paseo Tollocan, Universidad, 50130, Toluca City, State of Mexico, Mexico
${ }^{2}$ Interdisciplinary Research Laboratory Nanostructures and Biomaterials National School of Higher Education (ENES) Leon Unit, National Autonomous University of Mexico, Mexico Blvd. UNAM 2011, Los Tepetates, 37684, Leon Guanajuato, Mexico
${ }^{3}$ Research Center for Sustainable Chemistry Autonomus University of Mexico State, Km 14.5, Unidad San Cayetano, Toluca-Atlacomulco, 50200, Toluca City, State of Mexico, Mexico \\ Corresponding author, Rogelio J. SCOUGALL-VILCHIS; E-mail: rogelio_scougall@hotmail.com
}

\begin{abstract}
The surface roughness, morphology and shear bond strength (SBS) of dental zirconia using three different surface treatment techniques were evaluated. Three groups of sintered zirconia blocks were treated as follow, 1) Airborne-particle abrasion (APA) group (G1-APA), 50- $\mu \mathrm{m} \mathrm{Al}_{2} \mathrm{O}_{3} ; 2$ ) APA and 9\% hydrofluoric acid etching (G2-HF); 3) APA and Sodium Hydroxide (G3-NaOH). The specimens were evaluated for roughness [atomic force microscope (AFM)], morphology [Scanning Electron Microscope (SEM)] and for SBS in the universal testing machine. The AFM revealed changes in the roughness after the surface treatments, however there was not $R_{a}$ difference between groups, SEM analysis revealed changes in surface morphology for all surface treated specimens. For SBS, significant difference was found between G1-APA=8.4 $\pm 2.7 \mathrm{MPa}$ and $\mathrm{G} 2-\mathrm{HF}=3.3 \pm 0.6 \mathrm{MPa}(p<0.05)$ and $\mathrm{G} 2-\mathrm{HF}$ and $\mathrm{G} 3-\mathrm{NaOH}=9.0 \pm 3.0$ $\mathrm{MPa}(p<0.05)$. The main fracture mode was mixed failure $(63 \%)$ for G1-APA and G3-NaOH groups. G2-HF showed $100 \%$ adhesive failure. SBS was improved with $\mathrm{NaOH}$, however application of $\mathrm{HF}$ significantly decreased SBS.
\end{abstract}

Keywords: Zirconia, Adhesion, Surface treatment, Alkaline treatment, Acid treatment

\section{INTRODUCTION}

Due to non-silica based structure of zirconia, conventional techniques for adhesion of porcelains are not useful for this non silicate ceramics ${ }^{1}$. Therefore, the first attempts to improve resin and zirconia adhesion were directed in creating a silica cover on the surface of zirconia and then using the traditional adhesive system of silanation ${ }^{2}$. Silica coating of zirconia has been attempted through, silica tribochemical coating, silicon nitride hydrolysis ${ }^{3)}$, glazing porcelain on full sintered zirconia, use of silica slurry before sintering ${ }^{4)}$, in situ silica nanoparticle surface deposition ${ }^{5)}$, and glass bead cover before sintering ${ }^{6}$. Airborne-particle abrasion (APA) with $\mathrm{Al}_{2} \mathrm{O}_{3}$ and silica-coated aluminum of different granulometry are used as mechanical treatment ${ }^{7-10}$. Chemical surface treatment includes acid treatments, hydroxylation and monomers use. As hydrofluoric (HF) acid is not effective on zirconia degradation at conventional clinical concentrations at room temperature, different concentrations, temperature and/or exposure time have been tested, namely $40 \%$ concentration for $210 \mathrm{~s}^{11)}$, $48 \%$ concentration at $100^{\circ} \mathrm{C}$ for $25 \mathrm{~min}^{12)}, 9.5 \%$ concentration at $25^{\circ} \mathrm{C}$ for $1,2,3$ or $24 \mathrm{~h}$ or at $80^{\circ} \mathrm{C}$ for $1,3,5$ or $30 \mathrm{~min}$, and $48 \% \mathrm{HF}$ concentration at $25^{\circ} \mathrm{C}$ for 30 to $60 \mathrm{~min}^{13)}$. Another chemical approach is through surface treatment using monomers. It has been reported the effectiveness

Color figures can be viewed in the online issue, which is available at J-STAGE.

Received Mar 13, 2018: Accepted May 25, 2018

doi:10.4012/dmj.2018-078 JOI JST.JSTAGE/dmj/2018-078 of luting agents containing a hydrophobic phosphate monomer such as 10-methacryloyloxydecyl dihydrogen phosphate $(\mathrm{MDP})^{14)}$ after that report, the aim was to determinate whether or not it was consistent, leading to formulate, hypothesis regarding a chemical adhesion. In recent years chemical bonding evidence between zirconia surface and MDP monomer has been reported, because the presence of a chemical group of phosphate monomer (P)-O-Zr identified by time-of-flight secondary ion mass spectrometry after MDP surface treatment on zirconia $^{15)}$, while other research explained the chemical coupling mechanism between MDP and zirconia surface using a computational model, which showed that the coupling mechanism occurs in double-coordinate and single-coordinate configurations and that in the coupling process there are the possibility of an acid environment. Furthermore, they concluded that application of MDP in alkaline conditions improves shear bond strength $(\mathrm{SBS})^{16)}$. Regarding $\mathrm{pH}$ it has been reported that the use of MDP-based primers and alkaline solution contributed in increasing zirconia bond strength ${ }^{17)}$. Hydroxylation of bioinert zirconia surface was also proposed to increase the ${ }^{-} \mathrm{OH}$ groups so as to improve wettability and the reaction between surface and MDP resin cement monomers ${ }^{18)}$. However, few studies had been conducted regarding zirconia surface treatment using different $\mathrm{pH}$ solutions, for what the objective of this research was to evaluate the effect of three different surface treatments, namely mechanical and acid and alkaline solutions, 
on dental zirconia SBS, since the dual cured luting agents to adhere zirconia could be affected by the $\mathrm{pH}$ environment. The null hypothesis states that there are not SBS differences between three surface treatments to adhere zirconia.

\section{MATERIALS AND METHODS}

The brand, batch number and the composition of the materials used in this study are summarized in Table 1.

\section{Specimen preparation}

One-hundred fourteen zirconia square blocks, $(12.5 \times 12.5 \times 5 \mathrm{~mm})$ were cut from five presintered commercial dental zirconia discs (Zenostar, Wieland Dental+Technik, Pforzheim, Germany) in a CADCAM system (Zenotec Mini, Wieland Dental+Technik) using $1.0 \mathrm{~mm}$ carbide drills. Once manufactured, the blocks were sintered in a furnace (Zenotec Fire Cube, Wieland Dental+Technik) in standard set following the manufacturer instructions. The expected contraction was approximately $25 \%$, and the final zirconia block size was $10 \times 10 \times 4 \mathrm{~mm}$. The zirconia blocks were individually mounted in an autopolymerizing acrylic resin (Nic Tone, MDC Dental, Guadalajara, Mexico) in a custom-made tray with square-shaped molds $(2 \times 2 \times 1 \mathrm{~cm})$. Polymerizing was done while immersed in water to counter the exothermic effect. Ninety composite cylinders (Clearfil AP-X Esthetics, Kuraray Noritake Dental, Tokyo Japan), with a 5 -mm diameter and 2-mm thickness, were made in a nonstick matrix. Then, each side of the cylinder was light-cured with Bluephase N MC $800 \mathrm{~mW} / \mathrm{cm}^{2}$ (Ivoclar-Vivadent, Schaan, Liechtenstein) for $20 \mathrm{~s}$ as per manufacturer instructions -at $1 \mathrm{~mm}$ distance without touching the composite surface according to the lightcuring guideline ${ }^{19}$. Before adhesion process, composite cylinders were ultrasonically cleaned in $96 \%$ ethanol for $2 \mathrm{~min}$ and were dried at room temperature $\left(22-23 \pm 2^{\circ} \mathrm{C}\right)$. Zirconia samples were randomly assigned in three groups; 1) APA (G1-APA), 2) APA and HF acid (G2-HF), 3) APA and sodium hydroxide $(\mathrm{G} 3-\mathrm{NaOH}, \mathrm{gp} / \mathrm{n}=36)$ wherein $\mathrm{gp} / \mathrm{n}=30$ were for bond strength test, $\mathrm{gp} / \mathrm{n}=5$ for roughness measurement using atomic force microscope (AFM) and $\mathrm{gp} / \mathrm{n}=1$ for morphologic examination using scanning electron microscope (SEM). For the SBS, power value for the sample size in this study was calculated as post hoc using the highest and lowest mean value found for SBS and their respective standard deviations, and $\alpha=0.05$, on the basis of these values the power was $>0.80$.

\section{Surface treatment and adhesion procedure}

Zirconia blocks were treated by use of APA in a blaster (Eco Basic, Renfert, Hilzingen, Germany) with $50-\mu \mathrm{m}$ $\mathrm{Al}_{2} \mathrm{O}_{3}$ (Zeta Sand, Zhermack, RO, Italy) at a $90^{\circ}$ angle $10-\mathrm{mm}$ distance, 1 bar of pressure, $30 \mathrm{seg} / \mathrm{cm}^{2}$, and with a 1-mm diameter blaster tip. To standardize the procedure, a custom-made appliance was used to keep the distance, angle, and free movement across the samples, which were ultrasonically cleaned in $96 \%$ ethanol for $2 \mathrm{~min}$ and were dried with gentle oil-free air for $10 \mathrm{~s}$. For G1-APA, only APA treatment was applied.

Table 1 Comoposition, batch number, and manufacturers of materials used in the present study

\begin{tabular}{|c|c|c|c|}
\hline Material & Composition & Batch no. & Manufacturer \\
\hline Zeta Sand & $99.80 \% \mathrm{Al}_{2} \mathrm{O}_{3}$ & U112682/A & Zhermack Technical \\
\hline Zenostar MT & $\begin{array}{l}\text { Zirconium oxide }\left(\mathrm{ZrO}_{2}+\mathrm{HfO}_{2}+\mathrm{Y}_{2} \mathrm{O}_{3}\right) \geq 99.0 \% \\
\text { Ytrium oxide }\left(\mathrm{Y}_{2} \mathrm{O}_{3}\right)>6.5 \leq 8.0 \% \\
\text { Hafnium oxide }\left(\mathrm{HfO}_{2}\right) \leq 5 \% \text {, Aluminium oxide } \leq 1.0 \%\end{array}$ & U35562 & Wieland Dental \\
\hline Panavia F 2.0 & $\begin{array}{l}\text { A paste:10-methacryloyloxydecyl dihydrogen phosphate } \\
\text { (MDP), dl-Camphoroquinone, catalyst and iniciators; } \\
\text { paste B: hydrophobic aromatic dimethacrylate, barium glass } \\
\text { treated sodium fluoride, catalyst, accelerators and pigments }\end{array}$ & $\begin{array}{l}\text { 7D0107 } \\
\text { 7D0010 }\end{array}$ & Kuraray Noritake \\
\hline ED Primer II & $\begin{array}{l}\text { Liquid A: 2-hydroxyethyl methacrylate (HEMA), MDP } \\
\text { monomer, B: N-Methacryloyl-5-aminosalicylic acid, } \\
\text { accelerators and water. }\end{array}$ & $\begin{array}{l}\text { 7A0024 } \\
\text { 7A0144102023 }\end{array}$ & - \\
\hline $\begin{array}{l}\text { Clearfil Ceramic } \\
\text { Primer }\end{array}$ & $\begin{array}{l}\text { 3-Methacryloxypropyl trimethoxy silane, } \\
\text { MDP monomer and ethanol }\end{array}$ & 720011 & Kuraray Noritake \\
\hline $\begin{array}{l}\text { Clearfil AP-X } \\
\text { Esthetics }\end{array}$ & $\begin{array}{l}\text { Bisphenol-A-diglycidyl methacrylate (Bis-GMA) } \\
\text { Hydrophobic aromatic dimethacrylate, } \\
\text { dl-Camphorquinone, silanated barium glass filler } \\
\text { and pre-polymerized organic filler }\end{array}$ & 5CD143 & Kuraray Noritake \\
\hline Porcelain Etch & 9\% Hydrofluoric Acid & BCG4P & Ultradent \\
\hline Silane & Silane & BBY3G & Ultradent \\
\hline Sodium Hydroxide & $\mathrm{NaOH} \mathrm{99 \%}$ & 144102 & Fermont \\
\hline
\end{tabular}


For G2-HF, after APA the surface was treated with $9 \%$ HF acid, (Porcelain Etch, Ultradent Products, South Jordan, UT, USA) for 2 min, the HF was removed with water spray and was then ultrasonically cleaned as mentioned above. Before adhesion, a generous quantity of silane was applied in the surface and was left to dry for 2 min (Silane, Ultradent Products). For G3-NaOH, after the APA procedure, a $0.01-\mathrm{M} \mathrm{NaOH}$ solution (Sodium Hydroxide, CAS-1310-73-2, Fermont, Monterrey, Mexico) with a $\mathrm{pH}$ of 13.5 was generously applied and was left to dry for $10 \mathrm{~min}$ prior to adhesion as previously reported $^{16)}$. For all groups, a ceramic primer containing MDP-10 was applied generously (Clearfil Ceramic Primer, Kuraray Noritake Dental) for $5 \mathrm{~s}$ and left to dry for $40 \mathrm{~s}$. Composite cylinders were also conditioned as per manufacturer instruction. Dual curing cement (Panavia F 2.0, Kuraray Noritake Dental) was mixed and applied at zirconia surface. The composite cylinder was positioned on the zirconia surface using cotton pliers, and afterwards, a light finger pressure was applied for $10 \mathrm{~s}$. The excess cement was removed with a microbrush, and the oxygen-blocking agent was applied (Oxyguard, Kuraray Noritake Dental). After $5 \mathrm{~min}$, it was removed with gentle water spray. All the specimens were prepared by the same operator at room temperature $\left(22-23 \pm 2^{\circ} \mathrm{C}\right)$. The samples were stored in deionized water $\left(37 \pm 2^{\circ} \mathrm{C}\right)$ immediately after completion of the bonding procedure for $24 \mathrm{~h}$ and before SBS testing.

\section{Surface roughness and morphology}

After the surface treatment, five randomly selected samples from each experimental group and one control group $(\mathrm{G} 0 / \mathrm{n}=5)$ were evaluated through AFM (NaioAFM, Nanosurf, Liestal Switzerland). A $50 \times 50 \mu \mathrm{m}$ area was measured in tapping mode to determine the average roughness $\left(R_{\mathrm{a}}\right)$. Six measurements were taken from each sample, thus giving a total of 30 measurements per group. For morphologic examination, the representative samples in each group and one control sample $(n=1)$ were observed with an SEM (JEOL, JSM-6510LV, Tokyo, Japan) and after gold sputtering, the examination was conducted at $20-\mathrm{kV}$ acceleration and 2,000x magnification. For surface roughness the data were analyzed using a statistical program (SPSS 22.0 IBM, Chicago, IL, USA). Thereafter, descriptive statistics was obtained, the results were tested for normal distribution using the Shapiro-Wilk method. Kruskal-Wallis test, was carried out, and U-Mann-Whitney, as pairwise post hoc analysis, was performed after the data failed the test for normal distribution.

\section{SBS test and fracture mode}

The samples were immediately tested for SBS after being removed from water, in a universal testing machine (Autograph AGS-X, Shimadzu, Kyoto Japan), and great caution was enforced so as not to apply any force to the adhesive area during mounting in the apparatus. The test was performed at $1 \mathrm{~mm} / \mathrm{min}$ crosshead speed, and the debonding load was registered in MPa. After SBS test, all specimens were examined in a stereoscopic microscope at $40 \times$ magnification by a single observer to determine the failure mode, which was classified with the modified adhesive remnant index (ARI) criteria in which: Score $0=\mathrm{No}$ adhesive left on zirconia bonding surface (adhesive failure); Score 1=Less than half of the adhesive is left on zirconia bonding surface; Score $2=$ More than half of the adhesive is left on zirconia bonding surface; and Score $3=$ All adhesive is left on zirconia bonding surface ${ }^{20}$. Mixed failure for 1-2 scores and cohesive failure for score 3 . Thereafter, descriptive statistics was obtained, the Shapiro-Wilk, KruskalWallis test, and U-Mann-Whitney was applied for SBS. Moreover, statistical differences were investigated for ARI score by Chi-square test.

\section{RESULTS}

Roughness values $\left(\mathrm{R}_{\mathrm{a}}\right)$ measured with AFM (in $\mu \mathrm{m}$ ) and group comparison are shown in Fig. 1. Mean values per group are as follows: $\mathrm{G} 0=0.20 \pm 0.07 \mu \mathrm{m}, \mathrm{G} 1$ $\mathrm{APA}=0.29 \pm 0.01 \mu \mathrm{m}, \mathrm{G} 2-\mathrm{HF}=0.27 \pm 0.09 \mu \mathrm{m}$, and $\mathrm{G} 3-$ $\mathrm{NaOH}=0.28 \pm 0.11 \mu \mathrm{m}$. The values of experimental groups are comparable, with APA having the highest mean value. Kruskal-Wallis test revealed that the surface treatment had significant effect on surface roughness $(p<0.05)$. U-Mann-Whiney test revealed significant differences between control group and all experimental groups $(p<0.05)$. However, no significant difference was observed between APA group and chemical treatment groups G1-APA; G2-HF ( $p=0.779)$; G1-APA; G3-NaOH $(p=0.824)$; and $\mathrm{G} 2-\mathrm{HF}$; $\mathrm{G} 3-\mathrm{NaOH} \quad(p=0.842)$, which suggests that the chemical treatment has no significant effect on surface roughness.

The surface morphology micrographs are presented in Fig. 2, AFM surface micrographs show peaks and valleys that can be observed in experimental groups, whereas the crests and valleys observed in control group

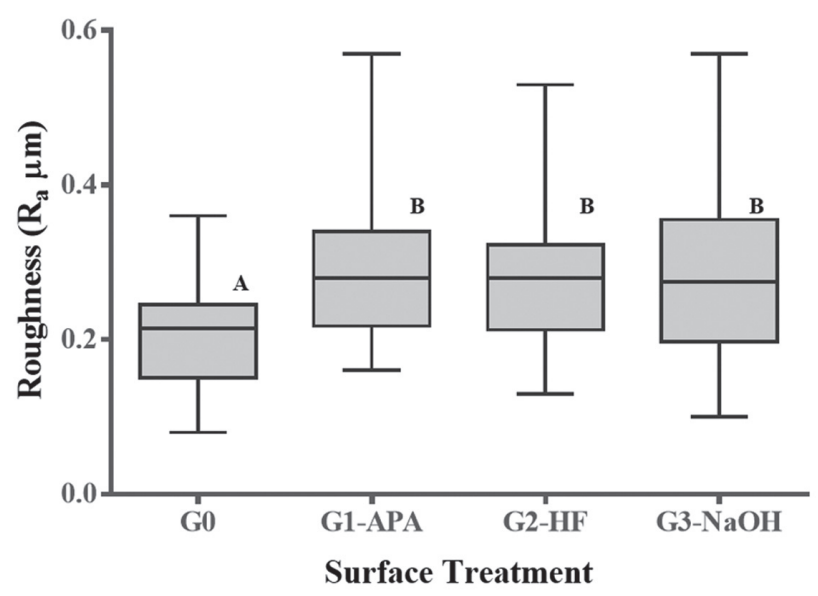

Fig. 1 Roughness comparison between groups; G0: control; G1-APA: APA; G2-HF; APA $/ 9 \% \mathrm{HF}$ and G3-NaOH: APA/NaOH through AFM.

U-Mann-Whitney's test $(p<0.05)$ different letters are significantly different groups. 

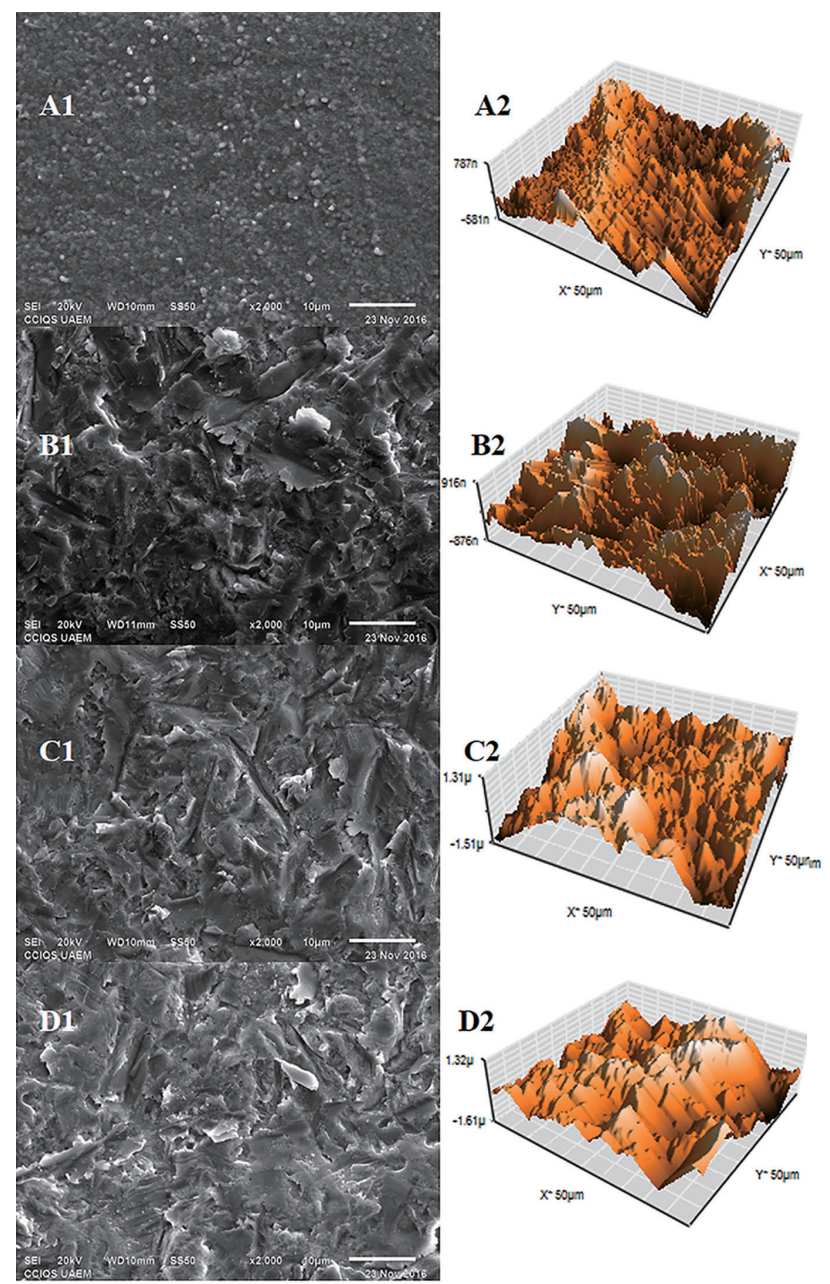

Fig. 2 Surface morphology after surface treatment, $\mathrm{SEM} \times 2,000$ magnification, $10 \mu \mathrm{m}$ bar (left) and AFM (right) micrographs.

A1-A2: control; B1-B2: APA; C1-C2: APA/ Hydrofluoric Acid; D1-D2: APA/Sodium Hydroxide. are lower and more homogeneous. SEM micrographs exhibited similar morphology for experimental groups; however, G1-APA showed more surface irregularities than the other groups. GC exhibited a regular surface, and neither scratches nor cracks were observed in any group. AFM and SEM micrographs are congruent with $R_{\mathrm{a}}$ values.

SBS values and the comparison between groups are shown in Table 2. The highest mean value belongs to the $\mathrm{G} 3-\mathrm{NaOH}$ surface treatment, followed by G1APA and the lowest mean value belongs to G2-HF. Nonparametric test Kruskal-Wallis revealed the effect of surface treatments on SBS of zirconia $(p<0.05)$ and U-Mann-Whitney test showed differences between experimental groups.

The distribution frequency and percentages score for failure mode after debonding are shown in Table 3. The Chi-Square comparison for ARI scores between groups indicated that the groups were not all the same (Chi-square $=22.46, p<0.001$ ). The predominant for G1APA and $\mathrm{G} 3-\mathrm{NaOH}$ reveals mixed failure. For G2-HF treatment, all the samples have an adhesive failure. The score of 3 was not found for any group, Table 4 .

Table 2 Mean and standard deviation (SD) for SBS (MPa) after $24 \mathrm{~h}$ water storage and Kruskal-Wallis analysis

\begin{tabular}{cc}
\hline Surface treatment & SBS \\
\hline G1-APA & $8.4^{\mathrm{A}}(2.7)$ \\
G2-HF & $3.3^{\mathrm{B}}(0.6)$ \\
G3-NaOH & $9.0^{\mathrm{A}}(3.0)$ \\
\hline
\end{tabular}

Mean values represented with different superscript uppercase letters are significantly different according to U-Mann-Whitney's test $(p<0.05)$

Table 3 Distribution, frequency and percentage of modified ARI scores

\begin{tabular}{|c|c|c|c|c|}
\hline Surface treatment & $0(\%)$ & $1(\%)$ & $2(\%)$ & Total \\
\hline G1-APA & $4(13.3)$ & $19(63.3)$ & 7 (23.3) & 30 \\
\hline $\mathrm{G} 2-\mathrm{HF}$ & $30(100)$ & $0(0)$ & $0(0)$ & 30 \\
\hline G3-NaOH & $9(30)$ & $19(63.3)$ & $2(6.6)$ & 30 \\
\hline Total & $43(47.7)$ & $38(42.2)$ & $9(10)$ & 90 \\
\hline
\end{tabular}

Score 0: Adhesive failure; 1 y 2: Mixed failure. Chi-square/Fisher's test $(p<0.001)$

Table 4 Failure mode by surface treatment group

\begin{tabular}{lccr}
\hline Group & Adhesive & Cohesive & Mixed \\
G1-APA & 4 & 0 & 26 \\
G2-HF & 30 & 0 & 0 \\
G3-NaOH & 9 & 0 & 21 \\
Total & 43 & 0 & 47 \\
\hline
\end{tabular}




\section{DISCUSSION}

The main objective of this study was to investigate the effect of three different surface treatments on SBS of dental zirconia resin cement interface, based on $\mathrm{pH}$ differences in each technique, and the null hypothesis states that there are not SBS differences between three surface treatments to adhere zirconia. The null hypothesis was rejected in this study, since there were found significant differences between groups.

Even several techniques for zirconia adhesion has been investigated, after a meta-analysis report that focused on zirconia adhesion, the authors stated that data reporting on zirconia treatments requires more standardization as it is of great importance to scientists gathering evidences regarding this matter ${ }^{21}$. Most of reported studies in vitro used slow-cut blade instruments followed by polishing zirconia surface before the adhesion procedure $\mathrm{e}^{3-6,8-10)}$. The common process of zirconia restorations in clinical practice is through computer aided design-computer aided manufacture (CAD-CAM), and the zirconia specimens used in this study were manufactured using the mentioned technology, which let us have a real approach regarding the roughness effect of the surface treatments in zirconia, as we measured the roughness on zirconia surface as it has been manufactured using CAD/CAM and after undergo to surface treatment. An important predicting variable to improve the SBS is the surface roughness. In previous studies some authors concluded that the APA of zirconia surface before the bonding procedure could be a key in success regardless of the particle granulometry ${ }^{10)}$. On the other hand, it has been published that there is a significant difference in surface roughness with respect to distance, and that there is significant difference as well in adhesive strength when the zirconia surface is treated by APA in different angles regardless of the distance ${ }^{8)}$. In this study, all experimental groups were treated by APA, and the findings of the previous researches, which specified the respective angles and distances for APA, and the instructions of the manufacturer regarding pressure and granulometry were all taken into account. In our research, the zirconia surface roughness of the control group, after it was designed and cut using the $\mathrm{CAD} / \mathrm{CAM}$ system, was reported, which could be a very important fact since the clinicians are getting zirconia inside surface restorations in similar conditions, so that the surface modification after surface treatment in this research was carried out close to the real dental practice. This study shows the roughness parameter $R_{a}$ through AFM measurement after different surface treatments namely combined mechanical and chemical treatment with an acid solution (HF 9\%) and an alkaline solution ( $\mathrm{NaOH}$ 0.01M, pH 13.5). Statistical analysis revealed that all experimental groups are significantly different with respect the control group, which is in line with the results from previous studies wherein researchers found that all APA specimens have shown a higher surface roughness than the control group after APA of the zirconia surface ${ }^{3,4,8)}$.
On the other hand, no difference was found between the experimental groups, and this can be due to the same mechanical surface treatment for all of them. Additional chemical treatments used in the study appear to have no effect on surface zirconia crystals degradation under the used protocol, and it consequently have no effect on surface roughness. There are some evidences that 9.5\% $\mathrm{HF}$ acid was able to modify the zirconia surface. However, the exposition time is at least $1 \mathrm{~h}$ immersion at $25^{\circ} \mathrm{C}$ or $1 \mathrm{~min}$ immersion at $80^{\circ} \mathrm{C}^{13)}$. In a similar study that is in line with our findings, for sodium hydroxide solution, no morphologic differences were found after the comparison of SEM micrographs of APA and acid and alkaline surface treatment ${ }^{16)}$. In this study observations of the surface with SEM showed that additional chemical treatment with acid or alkaline solution after APA did not alter substantially the surface morphology of all experimental groups, which is consistent with the computed $R_{a}$ values. As roughness is an important factor that promotes the mechanical interlocking between adhesive luting agent and zirconia surface, the nonexistence of difference between groups can be a good indicator that our results with respect to SBS are satisfactory. Is roughness a determinant of SBS or is it the chemical treatment in our study? For SBS, the lower value was computed for acid solution surface treatment, which have significant difference with those of APA and alkaline solution groups. The bond strength is greatly influenced by $50 \mu \mathrm{m}$ sized APA and in combination with MDP based product SBS achieved the highest mean, due to the APA treatment enhance the wettability and the MDP promotes the bond strength ${ }^{22)}$. Then if we have the same surface interlocking possibilities, and the chemical bonding by the MDP containing adhesive and resin cement we could suggest that under the same surface conditions in all groups, the results on SBS could be attributed to the effect of the chemical treatments acid and alkaline instead of the mechanical treatment. It can also be concluded that HF treatment have a negative effect on SBS under the conditions of our study, and these findings agree with a similar research in which the lowest mean value on their study was computed for the acid $\mathrm{pH}$ treatment group as compared with the neutral treatment group and alkaline surface treatment group $^{16)}$.

This can be attributed to formed P-O-Zr complex as the adhesive MDP-10 reacts with the zirconia surface ${ }^{15)}$, $\mathrm{H}^{+}$ion dissociation is necessary to give place to the $\mathrm{P}-\mathrm{O}$ $\mathrm{Zr}$ complex; and as a consequence, it has been suggested that high levels of $\mathrm{H}^{+}$could weaken the bond strength ${ }^{16)}$. The $\mathrm{pH}$ of adhesive systems influence bond strength of chemically cured resin materials, thus resulting in lower bond strength value for lower $\mathrm{pH}$ monomers, and this can be explained by the existing competition between residual acidic monomers and benzoyl peroxide for tertiary amine ${ }^{23)}$. In the other hand, even some evidences of chemical bonding between MDP monomer and zirconia surface has been reported ${ }^{15,16)}$, the mechanism is not fully explained. There are reported three possible mechanisms of MDP interaction with zirconia; hydrogen 
bonding, ionic bonding and both mechanisms in the same phosphate monomer ${ }^{24)}$, the authors propose that the MDP-zirconia interaction could occur in a neutral $\mathrm{pH}$ environment, as result only one $\mathrm{P}-\mathrm{OH}$ group can interact with either zirconia or neighboring phosphate group. In the current study a negative effect on SBS was found in the group with the acid surface treatment as mentioned previously. In this context, the mechanisms of acid environment in the MDP-zirconia reaction needs to be further researched, since the affinity to form hydrogen bonding of some acids with $\mathrm{ZrO}_{2}$ powder has been previously reported ${ }^{25)}$. A study proposed that an alkaline surface treatment can improve the SBS, in addition, found statistical significance while comparing neutral acid and alkaline $\mathrm{pH}$ groups, in which the alkaline surface treatment group ( $\mathrm{pH} 11-12)$ had the highest SBS even after 30 days of water storage ${ }^{16)}$. In our study the higher MPa value was computed for alkaline surface treatment group, but there was no significant difference in the SBS value when compared with APA group, and it is consistent with the results of a different study, where consisted in surface chemical treatment with primers containing MDP-10 monomer and $0.5 \mathrm{M}$ $\mathrm{NaOH}$ in single or combined treatments, and they found an improved SBS for groups that combined $\mathrm{NaOH}$ and MDP-10-containing primer. However, no significant differences were found in that study ${ }^{17)}$. Differences in the results of alkaline surface treatment between these researches can be attributed to the difference in the $\mathrm{pH}$ or $\mathrm{M}$ concentration in each of the studies, hence supporting the idea that SBS improvement could be $\mathrm{pH}$ value-dependent. However other research compared four different alkaline coatings with distinct $\mathrm{pH}$ values, and they concluded that higher alkaline $\mathrm{pH}$ values used before MDP conditioning would not necessarily improve bonding ${ }^{26)}$. Further studies should be made to determine a specific $\mathrm{pH}$ range to concisely improve the SBS as alkaline surface treatment could be a promising alternative to improve adhesion systems even in biological applications.

For ARI evaluation, we found that $63.3 \%$ of the fracture mode in APA and alkaline groups, belong to score 1 , which means that there is a cohesive failure within the resin cement material in some segment, suggesting that SBS between resin cement and zirconia is higher than the material itself. We also found a score of 0 for acid group that is equal to adhesive failure mode in all the samples, which means that there is no mechanical interlocking or chemical adhesion between zirconia and the luting agent.

In accordance with the results of this study, we can suggest that roughness and MDP monomers are not the only success key in zirconia adhesion, chemical surface treatment different to adhesive monomers also plays an important role in the process; we found that alkaline solution improves SBS values, while acid solutions have a negative effect. Meanwhile, acid solutions for surface treatments had been suggested for zirconia surface modification prior adhesion, it must be taken in account that zirconia luting agents are usually dual cure materials due to zirconia opacity and they have an initial chemical polymerizing process which could be affected by $\mathrm{pH}$.

\section{CONCLUSIONS}

Within the limitations of this in vitro study, the following conclusions can be drawn:

1. The surface treatment increased the roughness of zirconia and changed its morphology due to treatment with APA, HF acid, and sodium hydroxide.

2. The differences between treated surfaces do not have significant effects on surface roughness.

3. SBS was significantly affected by surface treatments with acid solution, whereas alkaline treatment with $\mathrm{NaOH}$ slightly improved SBS values.

4. The adhesive failure mode for HF group confirmed the negative effect of acid treatment on zirconia adhesion with dual cured resin cement.

\section{ACKNOWLEDGMENTS}

The authors thank to the Dental Technician Ana Maria Zavala Ruiz for her kind support in designing and manufacturing the zirconia specimens used in the present study in her dental laboratory facilities Vimars Soluciones Dentales ${ }^{\circledR}$.

\section{CONFLICT OF INTEREST}

Authors declare that they have no conflict of interest.

\section{REFERENCES}

1) Thompsom JY, Stoner BR, Piascik JR, Smith R. Adhesion/ cementation to zirconia and other non-silicate ceramics: Where are we now? Dent Mater 2011; 27: 71-82.

2) Piascik JR, Swift EJ. Critical appraisal. Resin bonding to zirconia. J Esthet Restor Dent 2012; 23: 417-420.

3) Lung CY, Liu D, Matinlinna JP. Silica coating of zirconia by silicon nitride hydrolysis on adhesion promotion of resin to zirconia. Mater Sci Eng C Mater Biol Appl 2015; 46: 103110.

4) Liu D, Pow EH, Tsoi JK, Matinlinna JP. Evaluation of four surface coating treatments for resin to zirconia bonding. $J$ Mech Behav Biomed Mater 2014; 32: 300-309.

5) Oliveira-Ogliari A, Collares FM, Feitosa VP, Sauro S, Ogliari FA, Moraes RR. Methacrylate bonding to zirconia by in situ silica nanoparticle surface deposition. Dent Mater 2015; 31: 68-76.

6) Martins AR, Gotti VB, Shimano MM, Borges GA, Gonçalves L de S. Improving adhesion between luting cement and zirconiabased ceramic with an alternative surface treatment. Braz Oral Res 2015; 29: http://dx.doi.org/10.1590/1807-3107BOR2015.vol29.0054.

7) Li R, Zhou H, Wei W, Wang C, Sun YC, Gao P. Effects of mechanical and chemical pretreatments of zirconia or fiber posts on resin cement bonding. PLoS ONE 2015; 10: e0129690.

8) Ho BJ, Tsoi JK, Liu D, Lung CY, Wong HM, Matinlinna JP. Effects of sandblasting distance and angles on resin cement bonding to zirconia and titanium. Int J Adhes Adhes 2015; 
62: 25-31.

9) Sciasci P, Abi-Rached FO, Adabo GL, Baldissara P, Fonseca RG. Effect of surface treatments on the shear bond strength of luting cements to Y-TZP ceramic. J Prosthet Dent 2015; 113: 212-219.

10) Gomes AL, Castillo-Oyagüe R, Lynch CD, Montero J, Albaladejo A. Influence of sandblasting granulometry and resin cement composition on microtensile bond strength to zirconia ceramic for dental prosthetic frameworks. J Dent 2013; 41: 31-41.

11) Menani LR, Farhat IA, Tiossi R, Ribeiro RF, Guastaldi AC. Effect of surface treatment on the bond strength between yttria partially stabilized zirconia ceramics and resin cement. J Prosthet Dent 2014; 122: 357-364.

12) Liu D, Tsoi JK, Mantinlinna JP, Wong HM. Effect of some chemical surface modifications on resin zirconia adhesion. $J$ Mech Behav Biomed Mater 2015; 46: 23-30.

13) Sriamporn T, Thamrongananskul N, Busabok C, Poolthong S, Uo M, Tagami J. Dental zirconia can be etched by hydrofluoric acid. Dent Mater J 2014; 33: 79-85.

14) Kern M, Wegner SM. Bonding to zirconia ceramic: adhesion methods and their durability. Dent Mater 1998; 14: 64-71.

15) Chen L, Suh BI, Brown D, Chen X. Bonding of primed zirconia ceramics: evidence of chemical bonding and improved bond strengths. Am J Dent 2012; 25: 103-108.

16) Xie H, Tray F, Zhang F, Lu Y, Shen S, Chen C. Coupling of 10-methacryloy loxydecyl dihydrogen phosphate to tetragonal zirconia: Effect of $\mathrm{pH}$ reaction conditions on coordinated bonding. Dent Mater 2015; 31: 218-225.

17) Lorenzoni FC, Leme VP, Santos LA, de Oliveira PC, Martins LM, Bonfante G. Evaluation of chemical treatment on zirconia surface with two primer agents and an alkaline solution on bond strength. Oper Dent 2012; 37: 625-633.

18) Lohbauer U, Zipperle M, Rischka R, Petschelf A, Fuller FA.
Hydroxylation of dental zirconia surface characterization and bonding potential. J Biomed Mater Res B Appl Biomater 2008; 87B: 461-467.

19) Roulet JF, Price R. Light curing-guidelines for practitioners. A consensus statement from the 2014 symposium on light curing in dentistry held at Dalhousie University, Halifax, Canada. J Adhes Dent 2014; 16: 303-304.

20) Artun J, Bergland S. Clinical trials with crystal growth conditioning as an alternative to acid-etch enamel pretreatment. Am J Orthod 1984; 85: 333-340.

21) Öscan M, Bernasconi M. Adhesion to zirconia used for dental restortions: a systematic review and meta-analysis. J Adhes Dent 2015; 17: 7-26.

22) Ahn JS, Yi YA, Lee Y, Seo DG. Shear bond strength of MDPcontaining self-adhesive resin cement and Y-TZP ceramics: Effect of phosphate monomer-containing primers. Biomed Res Int 2015; 2015: 389234.

23) Franco EB, Lopes LG, D’Alpino PH, Pereira JC. Influence of $\mathrm{pH}$ of different adhesive systems on the polymerization of chemically cured composite resin. Braz Dent J 2005; 16: 107111.

24) Nagaoka N, Yoshihara K, Feitosa VP, Tamada Y, Irie M, Yoshida Y, Van Meerbeek B, Hayakawa S. Chemical interaction mechanism of 10-MDP with zirconia. Sci Rep 2017; 7: 45563.

25) Pawsey S, McCormick M, De Paul S, Graf R, Lee YS, Reven L, Spiess HW. 1H Fast MAS NMR studies of hydrogen-bonding interactions in self-assembled monolayers. J Am Chem Soc 2003; 125: 4174-4184.

26) Qian M, Lu Z, Chen C, Zhang H, Xie H. Alkaline nanoparticle coatings improve resin bonding of 10-methacryloyloxydecyldi hydrogenphosphate-conditioned zirconia. Int J Nanomedicine 2016; 11: 5057-5066 